\title{
Détection des vulnérabilités psychiques en cancérologie
}

\section{Detection of psychological vulnerability in oncology}
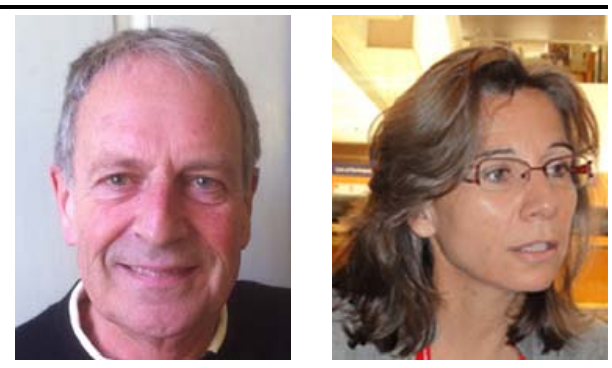

\author{
P. Guex - S. Dolbeault \\ (C) Springer-Verlag France 2013
}

Pour la psycho-oncologie, un des objectifs du troisième Plan cancer, attendu pour le 4 février 2014 et dont les lignes directrices sont parues en juillet dernier, est de répondre aux besoins des patients en souffrance psychique.

Les recommandations proposent d'intégrer systématiquement l'évaluation psychologique à la consultation d'annonce ou de la pratiquer dès que possible après le diagnostic, pour le patient et ses proches. Selon les besoins identifiés, une prise en charge psychologique doit être prévue dans le programme personnalisé de soins. Cela suppose évidemment que les ressources nécessaires soient mises à disposition.

Cette directive est conforme aux guides de bonnes pratiques du NCCN (National Comprehensive Cancer Network, 1999, 2011) qui ont inspiré le rapport de 1'IOM (Institute of Medicine) « Cancer care for the whole patient, meeting psychosocial health needs » («Soins cancérologiques pour le malade pris dans l'ensemble de sa personne : tenir compte de ses besoins psychosociaux »).

On ne revient pas ici sur le souci de déstigmatisation psychiatrique attaché au terme de détresse qui en est issu, conçu comme un continuum allant des sentiments normaux de vulnérabilité, tristesse et craintes face au cancer jusqu'à la crise psychique la plus grave nécessitant des soins psychiatriques spécialisés. Citons pour exemple le travail collectif de nombreux organismes tels qu'ASCO (American Society of Cli-

\section{P. Guex $(\square)$}

Faculté de biologie et de médecine, CHUV,

CH-1011 Lausanne, Suisse

e-mail : Patrice.Guex@chuv.ch

\section{S. Dolbeault $(\bowtie)$}

Département interdisciplinaire de soins de support pour

le patient en oncologie, institut Curie,

26, rue d'Ulm, F-75246 Paris cedex 05, France

e-mail : sylvie.dolbeault@free.fr

Inserm U699, Paris, France

Université Paris-Sud et université Paris-Descartes, UMR-S0669, Paris, France nical Oncology), IPOS (International Psycho-Oncology Society) ou APOS (American Psycho-Oncology Society), ACS (American College of Surgeons), QOPI (Quality Oncology Practice Initiative), pour défendre la science du soin et des standards psychosociaux en oncologie.

Le thermomètre de la détresse DT (distress thermometer, en fait une EVA mesurant le désormais sixième signe vital oncologique que constitue la détresse) a été validé dans différentes situations oncologiques et présente une très bonne concordance avec l'échelle d'anxiété-dépression HADS (Hospitalized Anxiety and Depression Scale) utilisée en psychiatrie.

Le score de DT est articulé avec un algorithme décisionnel permettant de planifier l'orientation du patient en fonction de ses besoins, de sa souffrance ou de sa psychopathologie.

De nombreuses crises jalonnent en effet le parcours du patient, autour du temps diagnostique, des traitements, de la « survie » ou de la rechute par exemple. Ce sont autant d'étapes de plus grande vulnérabilité pour lui et pour son entourage sociofamilial, où les besoins et les «détresses » doivent être réévalués. Ces derniers se modifient et évoluent en effet tout au long de ce parcours de soins et sont souvent mal reconnus par les professionnels du soin. Cette vulnérabilité constitue une source supplémentaire de morbidité psychique et physique, de mauvaise qualité de vie ou de survenue de handicaps d'autre nature.

$\mathrm{Au}$ fil des années, la Société française de psychooncologie (SFPO), aussi bien par ses interventions itératives auprès des autorités que - plus récemment — par l'élaboration de recommandations de bonnes pratiques cliniques, a édicté des standards de qualité visant le repérage précoce des vulnérabilités psychologiques, psychosociales ou psychiatriques des patients et des familles en cancérologie. Ces guides diagnostiques et de bonnes pratiques sont des précurseurs essentiels du développement et de l'amélioration des prises en soins ainsi que de la compétence de tous les professionnels dans le domaine.

Le sommaire de ce numéro réunit les contributions de plusieurs auteures françaises actives dans des centres 
anticancéreux ou universitaires, qui s'inscrivent complètement dans cette stratégie de meilleure détection et de traitements garantis. Deux articles en anglais, venus respectivement d'Italie et des Pays-Bas, ouvrent quant à eux le vaste débat sur la pertinence comparée des outils diagnostiques et des échelles psychométriques validées, construites par consensus.

Isabelle Piollet aborde la question importante de la confusion, le delirium, complication neuropsychiatrique la plus courante en cancérologie. Régulièrement sousdiagnostiquée et de ce fait non traitée, elle résulte d'une combinaison de facteurs de risque liés à l'âge et à la fragilité somatopsychique préexistante ; cela conduit à une morbidité et à une mortalité accrues interférant avec le contrôle des symptômes, les durées d'hospitalisation, l'émergence d'une détresse et la souffrance des patients et des équipes. Ce texte représente une des premières recommandations de la SFPO.

Isabelle Léger et al. décrivent la consultation mémoire de l'institut Gustave-Roussy (IGR) et l'évaluation, essentielle, des troubles cognitifs survenant au moment du diagnostic ou lors des traitements, s'appuyant sur des compétences neuropsychologiques spécifiques. Ces ressources encore trop peu répandues permettent de distinguer les troubles cognitifs vrais, largement sous-évalués en consultation, des réactions émotionnelles et subjectives.

La dépression est fréquente à tous les stades en cancérologie, elle constitue un facteur de risque suicidaire de même qu'une cause importante de non-adhésion aux traitements oncologiques. Il est essentiel d'en évaluer la nature et l'intensité, en particulier la frontière entre la détresse et les épisodes dépressifs plus graves nécessitant des traitements spécialisés. C'est la contribution de Sarah Dauchy et al. de faire l'inventaire des troubles dépressifs et de leurs traitements. Ce texte constitue aussi une recommandation de la SFPO.

Marie-Frédérique Bacqué part de ses recherches réalisées à Strasbourg sur le deuil pour le resituer en tant que processus psychodynamique de la personne, mais aussi comme facteur de vulnérabilité. Elle le distingue clairement de la dépression dans la trajectoire de la maladie cancéreuse, ce qui n'exclut pas que des formes de deuils compliqués produisent des tableaux dépressifs graves, surtout chez des personnes prédisposées.

Cette différenciation entre processus subjectifs, réactions psychologiques au cancer et à ses traitements et les systèmes nosologiques psychiatriques multiaxiaux types DSM, ICD est très bien développée par Luigi Grassi et al. L'école italienne de Ferrara travaille depuis longtemps sur des critères diagnostiques pour la recherche en psychosomatique (DCPR), suivant la tradition biopsychosociale. Dans le champ du cancer, le DCPR permet de rendre compte des « comportements maladies » mais aussi des symptômes somatiques fonctionnels se superposant aux troubles liés directement au cancer.
Cette recherche a influencé l'introduction de nouvelles entrées dans le DSM 5 récemment paru, sous le chapitre « Facteurs psychologiques affectant d'autres atteintes médicales ».

Enfin, James. C. Coyne conclut ce florilège par ses réflexions sophistiquées sur les limites de l'introduction d'une évaluation en routine de la détresse en cancérologie. Il considère les choses sous les différents angles de la santé publique, de l'épidémiologie, des outils psychométriques eux-mêmes et de leurs biais, des effets sur la clinique (out$p u t)$ et plus globalement sur la satisfaction générale du patient ainsi que sur sa qualité de vie (outcome); enfin, sous l'angle des conséquences pour les institutions et des coûts de la santé du repérage de paramètres par des (bio)marqueurs, ici des (psycho)marqueurs qui amélioreraient peu l'évolution des malades ou qu'on ne pourrait considérer, faute de ressources financières et professionnelles appropriées.

Certaines positions marquées de J. Coyne (connu dans la communauté psycho-oncologique pour ses positions critiques souvent radicales !) peuvent apparaître en contradiction avec certaines recommandations de bonnes pratiques cliniques présentées dans les divers articles de ce numéro spécial. Sachons avant tout garder une vue d'ensemble et porter un regard ouvert sur l'évolution de l'état de la connaissance dans ce champ en plein développement, qui soulève aujourd'hui autant de questions qu'il n'en résout.

Rappelons l'état des connaissances en 2013 : la démarche de repérage systématique de la détresse auprès des patients atteints de cancer a déjà démontré deux choses de manière certaine : d'une part, son intérêt en termes d'amélioration de la communication entre le soignant et le soigné, d'autre part, son influence positive sur la capacité du soignant à orienter vers l'expert en santé mentale les patients relevant d'une situation de détresse clinique significative.

La question de savoir si cette organisation, certes contraignante et coûteuse, d'un screening systématique de la détresse entraîne une amélioration directe de l'état psychique du patient reste l'objet du débat. Réjouissons-nous de voir le nombre d'équipes de cliniciens et de chercheurs en psycho-oncologie qui tentent d'affiner notre connaissance du domaine, tout en rappelant en permanence que l'enjeu de ce débat sous-tend et sous-tendra toujours la question de la capacité des professionnels à être en lien avec les personnes qu'ils soignent.

Dans le dernier numéro d'octobre de la Revue médicale suisse, le patron de la médecine interne lausannoise, le Pr G. Waeber, intitulait son éditorial «Internistes généralistes et biomarqueurs » pour conseiller finalement de relativiser les trop nombreux biomarqueurs à l'utilité parfois discutable au profit d'un engagement massif en faveur de la formation de bons cliniciens, garantissant la relève en médecine interne générale en particulier.

Pour la psycho-oncologie, spécialité encore en quête de légitimité scientifique, l'un n'exclut peut-être pas l'autre, psychométrie et excellence clinique! 